\title{
How many times can a function be iterated?
}

\author{
Massimo Gobbino \\ Università degli Studi di Pisa \\ Dipartimento di Matematica Applicata "Ulisse Dini" \\ Via Filippo Buonarroti 1c, 56127 PISA, Italy \\ e-mail: m.gobbino@dma.unipi.it \\ Robert Samuel Simon \\ London School of Economics \\ Department of Mathematics \\ Houghton Street, WC2A 2AE, London, United Kingdom \\ e-mail: R.S.Simon@lse.ac.uk
}




\begin{abstract}
Let $C$ be a closed subset of a topological space $X$, and let $f: C \rightarrow X$. Let us assume that $f$ is continuous and $f(x) \in C$ for every $x \in \partial C$.

How many times can one iterate $f$ ?

This paper provides estimates on the number of iterations and examples of their optimality. In particular we show how some topological properties of $f, C, X$ are related to the maximal number of iterations, both in the case of functions and in the more general case of set-valued maps.

We also show how this problem is related to the existence of equilibria for stochastic games.
\end{abstract}

Mathematics Subject Classification 2000 (MSC2000): 54H20, 37B99, 55N05.

Key words: Viability Theory, Dynamic Systems, iteration of functions, Point-Set Topology, Cech-Alexander Cohomology. 


\section{Introduction}

Let $C$ be a closed subset of a topological space $X$, and let $f: C \rightarrow X$. We investigate the existence of finite or infinite sequences (orbits) $\left\{x_{i}\right\}_{i \in I}$ in $X$, where $I=\{0,1, \ldots, n\}$ or $I=\mathbb{N}$, such that $x_{i}=f\left(x_{i-1}\right)$ for every $i \in I$ with $i \geq 1$.

At this level of generality there is of course no reason for such a sequence to exist with $n>1$. For this reason we assume two conditions on $f$ :

- $f$ is continuous;

- $f$ maps $\partial C$ back to $C$, namely $f(x) \in C$ for every $x \in \partial C$.

For want of a better term, we call this topic "Discrete Viability Theory". Surprisingly this problem seems to be quite new. Up to now indeed we have found little related literature, although this topic seems to come close to different areas. Let us mention some of them.

- Conventional Viability Theory. This theory, for which we refer the reader to J. P. Aubin [1], considers continuous-time dynamic processes with some control mechanism. The main problem is finding conditions under which these processes stay within a given set $C$. As in our problem, these conditions often involve the behavior of the flow at the boundary of $C$. Unfortunately there are relatively few theorems for discrete-time models: one example is Theorem 3.7.11 of [1] where $X=\mathbb{R}^{n}, C$ is a convex subset, $f$ is a multi-valued map with convex images, and the existence of a fixed point is proven.

- Fixed point theorems. If $f$ has a fixed point $x \in C$, then we can clearly iterate $f$ infinitely many times starting from $x$. So the most interesting case is when $f$ has no fixed point. Let us assume however that an infinite orbit exists. Then under general assumptions the $\omega$-limit of this orbit is a closed $f$-invariant set, hence a fixed point of $f$ as a function acting on the space of closed sets. In this way the existence of an infinite orbit is reduced to a fixed point problem. Unfortunately up to now this approach didn't work because it's difficult to find topological obstructions in the space of closed subsets.

- Dynamical systems. On the one hand our problem can be considered as a problem in discrete-time dynamical systems or in topological dynamics. On the other hand, to our knowledge in all the literature the iterations are always well defined for the trivial reason that $C=X$, and the main questions concern their asymptotic behavior. Continuous-time dynamical systems are weakly related to our topic if we add the assumption that $f$ is homotopic to the identity. In this case indeed $f(x)$ could be interpreted as the position at time $t=1$ of a continuous trajectory which starts from $x$ at time $t=0$. If this is the case we could apply the classical 
tools for the study of flows, such as for example the Conley Index Theory (see [5]). However in general there is no flow which connects $x$ and $f(x)$, and for this reason we dismissed this approach as hopeless. One could argue that the construction known as "suspension of a map" (or "the mapping torus") describes how to turn a map on a space $X$ into a flow on a different topological space $Y$, but this could at most provide trajectories in $Y$.

- Game Theory. One of the main problems in game theory is the existence of equilibria. Classical results in this field are usually proved by means of fixed point theorems (see for example the celebrated result by J. NASH [6]). In Section [5 we show that there are special stochastic games called quitting games for which the existence of approximate equilibria is equivalent to the existence of suitable non-stationary orbits for some multi-valued functions. Unfortunately the counterexamples we present in this paper, one of which (Example 4.9) was inspired by a game theoretic context, show that this equivalence doesn't lead to a simple proof of existence of equilibria.

Let us come now to an explicit example of a question. Let us consider the case where $X=\mathbb{R}$, and $C$ is an interval. Our assumption on the behavior of $f$ at the boundary implies that $f(0) \geq 0$ and $f(1) \leq 1$, hence $f$ admits a fixed point because of the Intermediate Value Theorem. Therefore the simplest nontrivial problem in Discrete Viability Theory is probably the following one.

Problem 1.1 Let $C$ be a compact and connected subset of $X=\mathbb{R}^{n}$. Let $f: C \rightarrow X=$ $\mathbb{R}^{n}$ be a continuous function such that $f(x) \in C$ for every $x \in \partial C$.

How many times can we iterate $f$ starting from a suitable $x \in C$ ?

What motivates us the most is the search for some theorem that implies the existence of an infinite orbit for functions that neither have fixed points nor $f(C) \subseteq C$. When we started attacking this problem we were rather optimistic about the existence of such a theorem under general assumptions on $f, C, X$, and all the colleagues we contacted in that period shared our optimism. The first two iterations are indeed given for free, and a simple connectedness argument provides two more iterations. At a first glance it seemed also possible to reiterate the argument (see Remark 2.6) assuming only the connectedness of $X$ and $C$.

Our optimism decreased when T. WiandT [15] showed us a simple situation (see Example 4.3) where $X$ and $C$ are compact and connected but only four iterations are possible. That example showed us that further requirements on $f, C, X$ were needed in order to perform further iterations of $f$. In order to rule out the situation of Example 4.3 we worked in two different directions: either by asking that $f$ is homotopic to the identity in a suitable sense (see Problem 2.2), or by requiring $X$ to be simply connected, since in that example $X$ is the unit circle $S^{1}$. In both cases we succeeded in 
proving the existence of a fifth iteration (Theorem 2.7. Theorem 2.8 and Theorem 2.12). However, the argument is more involved, and surprisingly more or less the same despite of the different additional assumptions.

The little optimism left become pessimism when we found Example 4.6, where $f$, $C, X=\mathbb{R}^{2}$ are as in Problem 1.1, $f$ is homotopic to the identity in the suitable sense, and nevertheless only six iterations can be computed.

In any case we are not sure that this is the end of the story, because probably further topological requirements on $C$ can provide more iterations (see Section 6). The space $\mathbb{R}^{2}$ is very restrictive concerning the topological options for a subset $C$ that is the closure of an open set. It is conceivable that some additional conditions that would imply the existence of an infinite orbit (but not necessarily a fixed point) in higher dimensions would imply the existence of a fixed point in the $X=\mathbb{R}^{2}$ context.

In this paper we present some lower bounds for the number of iterations in terms of the topological properties of $f, C, X$, and we show their optimality with some examples. In order to give a complete theory we work both with functions and with set-valued maps (a good reference on iterating set-valued maps is [4]). Many parts of the theory are similar in both cases, but there are also some remarkable differences (see Remark 2.13).

This paper is organized as follows. In Section 2 we state the questions and our results. In Section 3 we prove the results. In Section 4 we present some examples showing the optimality of our estimates. In Section 5 we present the connections with game theory which motivated this study. In Section [6 we state some open problems.

\section{Statements}

Throughout this paper, unless otherwise stated, $X$ denotes a topological space. Given $A \subseteq X, \operatorname{Int}(A)$ denotes the set of interior points, $\operatorname{Clos}(A)$ the closure, $\partial A$ the boundary of $A$ in $X$. We recall that $X$ is said to be locally connected if every $x \in X$ has a fundamental system of connected neighborhoods.

Every $Y \subseteq X$ may be regarded as a topological space itself, with the topology inherited as a subset of $X$. If now $A \subseteq Y$, then $\operatorname{Int}_{Y}(A), \operatorname{Clos}_{Y}(A), \partial_{Y} A$ denote, respectively, the set of interior points, the closure, and the boundary of $A$ relative to the topological space $Y$.

We say that $Y$ satisfies the fixed point property if every continuous function $g: Y \rightarrow$ $Y$ has a fixed point. For example, any nonempty compact convex subset of $\mathbb{R}^{n}$ has the fixed point property because of Brouwer's fixed point theorem.

In this paper we make a mild use of Cech-Alexander cohomology, in the sense that in some statements we assume that $\check{H}^{1}(X)=0$, namely that the first Cech-Alexander cohomology group (with $\mathbb{Z}$ as coefficient group, just to fix the ideas) is trivial. For readers which are not familiar with this cohomology theory, in Lemma 3.2 we show that for reasonable spaces (e.g. paracompact Hausdorff spaces) this assumption implies 
the following: "for every open set $A \subseteq X$, if $A$ and $X \backslash A$ are connected, then $\partial A$ is connected". This last property is what we use in this paper. We recall also that a simple case in which $\check{H}^{1}(X)=0$ is when $X$ is locally contractible and simply connected. Good references for Cech-Alexander cohomology are Chapter 3 of [3] and Chapter 6 of [11].

\subsection{DVT for functions}

The following is the main question in what we called Discrete Viability Theory.

Problem 2.1 Let $X$ be a topological space, and let $C \subseteq X$ be a nonempty closed subset. Let $f: C \rightarrow X$ be a continuous function such that $f(x) \in C$ for every $x \in \partial C$.

How many times can we iterate $f$ starting from a suitable $x \in C$ ?

In the following problem we strengthen the assumptions on $f$ by asking that $f$ is homotopically equivalent to the identity map on $C$ by a homotopy whose intermediate maps also send $\partial C$ back to the set $C$.

Problem 2.2 Let $X, C$, and $f$ be as in Problem [2.1. Let us assume that there exists a function $\Phi: C \times[0,1] \rightarrow X$ such that

- $\Phi(x, 0)=x$ for every $x \in C$;

- $\Phi(x, 1)=f(x)$ for every $x \in C$;

- $\Phi(x, t) \in C$ for every $x \in \partial C$ and every $t \in[0,1]$.

How many times can we iterate $f$ starting from a suitable $x \in C$ ?

In order to better investigate these problems, we introduce some notations.

Definition 2.3 Let $X, C$, and $f$ be as in Problem 2.1. We recursively define a sequence $\left\{C_{n}\right\}_{n \in \mathbb{N}}$ of subsets of $X$ by

$$
C_{0}:=X, \quad C_{n+1}:=\left\{x \in C: f(x) \in C_{n}\right\} .
$$

Then we set

$$
\begin{gathered}
A_{n}:=C_{n} \backslash C_{n+1} \\
\operatorname{Iter}(f, C, X):=\sup \left\{n \in \mathbb{N}: C_{n} \neq \emptyset\right\} \in \mathbb{N} \cup\{+\infty\} .
\end{gathered}
$$

The following proposition clarifies the set-theoretic properties of the notions we have just introduced (proofs are trivial). 
Proposition 2.4 Let $X$ be a set, let $C \subseteq X$ be a nonempty subset, and let $f: C \rightarrow X$ be any function.

Then the notions introduced in Definition 2.3 fulfil the following properties:

(1) Iter $(f, C, X)$ is the maximal length of a sequence $x_{0}, \ldots, x_{n}$ such that $x_{i}=f\left(x_{i-1}\right)$ for every $i=1, \ldots, n$;

(2) $C_{n+1} \subseteq C_{n}$ for every $n \in \mathbb{N}$;

(3) if $C_{n+1}=C_{n}$ for some $n \in \mathbb{N}$, then $C_{m}=C_{n}$ for every $m \geq n$;

(4) if $x \in C_{n+1}$ then $f(x) \in C_{n}$;

(5) if $x \in A_{n+1}$ then $f(x) \in A_{n}$;

(6) if $\operatorname{Iter}(f, C, X)=k<+\infty$, then $A_{i} \neq \emptyset$ if and only if $i \leq k$.

We state now the topological properties of the sets $A_{n}$ and $C_{n}$.

Proposition 2.5 Let $X, C$, and $f$ be as in Problem 2.1.

Then for every $n \in \mathbb{N}$ we have that (for simplicity we use $\partial_{n}$ instead of $\partial_{C_{n}}$ to denote boundaries relative to $C_{n}$ )

(1) $C_{n}$ is a closed subset of $X$;

(2) $f\left(\partial_{n+1} C_{n+2}\right) \subseteq \partial_{n} C_{n+1}$;

(3) $\partial_{n} C_{n+1} \subseteq C_{n+2}$;

(4) $A_{n} \cup C_{n+2}$ is a closed set.

Remark 2.6 As a consequence of Proposition 2.4 and Proposition 2.5, by restricting the domain and the codomain, we can regard $f$ as a function $f: C_{n+1} \rightarrow C_{n}$, and this restriction satisfies $f\left(\partial_{n} C_{n+1}\right) \subseteq C_{n+1}$. Therefore, if $f: C \rightarrow X$ satisfies the assumptions of Problem [2.1, then $f: C_{n+1} \rightarrow C_{n}$ satisfies the same assumptions for every $n \in \mathbb{N}$, and $\operatorname{Iter}(f, C, X)=n+\operatorname{Iter}\left(f, C_{n+1}, C_{n}\right)$.

If we know a priori that $C_{n}$ is connected for every $n \in \mathbb{N}$, this leads to an inductive proof that $C_{n} \neq \emptyset$ for every $n \in \mathbb{N}$. But we can find no non-trivial condition that forces this to hold, and simple examples can be given where infinite orbits (and also fixed points) exist and $C_{0}$ and $C_{1}$ are connected, but $C_{n}$ in not connected for all $n \geq 2$.

If $C_{n}$ is not connected it may happen that $C_{n+1}$ is the union of some connected components of $C_{n}$ : in this case $f$ can map $C_{n+1}$ into the remaining connected components of $C_{n}$, causing $C_{n+2}$ to be empty (see the examples in Section 4).

This points out once more the importance of relative boundaries in Proposition 2.5: boundaries are always defined relative to something, and that something can change at each step. 
The following result provides our estimates on the number of iterations for Problem 2.1.

Theorem 2.7 Let $X, C$, and $f$ be as in Problem 2.1, and let $\operatorname{Iter}(f, C, X)$ be as in Definition 2.3. Then we have the following estimates.

(1) If $\partial C \neq \emptyset$ then $\operatorname{Iter}(f, C, X) \geq 2$.

(2) If $X$ is connected, then $\operatorname{Iter}(f, C, X) \geq 3$.

(3) If $X$ is connected, and $C$ is connected, then $\operatorname{Iter}(f, C, X) \geq 4$.

(4) Let us assume that $C$ is connected, and that $X$ is a paracompact Hausdorff space which is connected, locally connected and satisfies $\check{H}^{1}(X)=0$.

Then $\operatorname{Iter}(f, C, X) \geq 5$.

(5) If $\partial C$ is a retract of $X \backslash \operatorname{Int}(C)$, and $C$ satisfies the fixed point property, then there exists $x \in C$ such that $f(x)=x$. In particular $\operatorname{Iter}(f, C, X)=+\infty$.

Under the assumptions of Problem 2.2 we have the following result (note that there are no topological requirements on $C$ and $X$ ).

Theorem 2.8 Let $X, C$, and $f$ be as in Problem 2.2, and let $\operatorname{Iter}(f, C, X)$ be as in Definition [2.3.

Then we have that $\operatorname{Iter}(f, C, X) \geq 5$.

Some examples in Section 4 show the optimality of these estimates.

\subsection{DVT for set-valued maps}

In this section we extend some parts of the theory from functions to set-valued maps. Let us begin with some notations and definitions.

Let $X$ be a topological space, let $C \subseteq X$ be a closed subset, and let $\mathcal{P}_{\star}(X)$ be the set of nonempty subsets of $X$. A set-valued map on $C$ with values in $X$ is any map $f: C \rightarrow \mathcal{P}_{\star}(X)$.

The first thing we need is some continuity of $f$. There are several notions of continuity for set-valued maps, and all of them are equivalent to standard continuity in the case of single-valued maps. The notion we use in this paper is usually referred in the literature as upper semicontinuity, and it is defined as follows.

(usc) A map $f: C \rightarrow \mathcal{P}_{\star}(X)$ is upper semicontinuous if for every open set $U \subseteq X$ we have that $\{x \in C: f(x) \subseteq U\}$ is an open subset of $C$. 
Then we need to control the behavior of $f$ at $\partial C$. The assumption in Problem 2.1 can be extended to set-valued maps in a weak and in a strong sense (equivalent if $f$ is single-valued), as follows.

(Bdr-w) For every $x \in \partial C$ we have that $f(x) \cap C \neq \emptyset$.

(Bdr-s) For every $x \in \partial C$ we have that $f(x) \subseteq C$.

Finally, simple examples (see Example 4.8) show that nothing but the trivial iterations can be expected without connectedness assumptions on the images. For this reason, we often need the following property (trivially satisfied by functions).

(Conn) For every $x \in C$ we have that $f(x)$ is connected.

We can now state the main question in Discrete Viability Theory for set-valued maps.

Problem 2.9 Let $X$ be a topological space, let $C \subseteq X$ be a nonempty closed subset, and let $f: C \rightarrow \mathcal{P}_{\star}(X)$ be a set-valued map satisfying (usc), (Bdr-w) or (Bdr-s), and (Conn).

How many times can we iterate $f$ starting from a suitable $x \in C$ ?

In order to study this problem, in analogy with the case of functions we consider the sequence of sets $\left\{C_{n}\right\}_{n \in \mathbb{N}}$ recursively defined by

$$
C_{0}:=X, \quad C_{n+1}:=\left\{x \in C: f(x) \cap C_{n} \neq \emptyset\right\},
$$

and then we define $A_{n}$ and $\operatorname{Iter}(f, C, X)$ as in Definition 2.3.

The set-theoretic properties of these notions are analogous to the case of functions. We sum them up in the following Proposition.

Proposition 2.10 Let $X$ be a set, let $C \subseteq X$ be a nonempty subset, and let $f: C \rightarrow$ $\mathcal{P}_{\star}(X)$.

Then statements (2), (3), (6) of Proposition 2.4 hold true without changes. Moreover, statements (1), (4), (5) of Proposition 2.4 hold true in the following modified form:

$\left(1^{\prime}\right) \operatorname{Iter}(f, C, X)$ is the maximal length of a sequence $x_{0}, \ldots, x_{n}$ such that $x_{i} \in f\left(x_{i-1}\right)$ for every $i=1, \ldots, n$;

(4) if $x \in C_{n+1}$ then $f(x) \cap C_{n} \neq \emptyset$;

(5) if $x \in A_{n+1}$ then $f(x) \subseteq A_{0} \cup \ldots \cup A_{n}$ and $f(x) \cap A_{n} \neq \emptyset$. 
The topological properties of the sets $A_{n}$ and $C_{n}$ are analogous to the case of functions only for small values of $n$, as stated in the following Proposition.

Proposition 2.11 Let $X$ be a topological space, let $C \subseteq X$ be a closed subset, and let $f: C \rightarrow \mathcal{P}_{\star}(X)$ be a set-valued map satisfying (usc), (Bdr-w) and (Conn).

Then (we use $\partial_{n}$ instead of $\partial_{C_{n}}$ to denote boundaries relative to $C_{n}$ )

(1) $C_{n}$ is a closed subset of $X$ for every $n \in \mathbb{N}$;

(2) $A_{0}$ and $A_{1}$ are open subsets of $X$;

(3) $\partial_{1} C_{2} \subseteq C_{3}$

(4) $A_{1} \cup C_{3}$ is a closed subset of $X$, hence $A_{0} \cup A_{2}$ is an open subset of $X$;

(5) $\partial_{2} C_{3} \subseteq C_{4}$;

(6) $A_{2} \cup C_{4}$ is a closed subset of $X$, hence $A_{0} \cup A_{1} \cup A_{3}$ is an open subset of $X$;

(7) if $x \in \partial_{3} C_{4} \cap A_{4}$ then $f(x) \cap A_{2} \neq \emptyset$ and $f(x) \cap A_{3} \neq \emptyset$.

The following result is the counterpart of Theorem 2.7 for set-valued maps.

Theorem 2.12 Let $X$ be a topological space, let $C \subseteq X$ be a closed subset, let $f: C \rightarrow$ $\mathcal{P}_{\star}(X)$ be a set-valued map, and let $\operatorname{Iter}(f, C, X)$ be as in Definition 2.3.

Then we have the following estimates.

(1) If $\partial C \neq \emptyset$ and $f$ satisfies (Bdr-w) then $\operatorname{Iter}(f, C, X) \geq 2$.

(2) Let us assume that $X$ is connected, and $f$ satisfies (usc), (Bdr-w), and (Conn). Then $\operatorname{Iter}(f, C, X) \geq 3$.

(3) Let us assume that $X$ is connected, $C$ is connected, and $f$ satisfies (usc), (Bdr-w), and (Conn). Then $\operatorname{Iter}(f, C, X) \geq 4$.

(4) Let us assume that

- $X$ is a paracompact Hausdorff space which is connected, locally connected and satisfies $\check{H}^{1}(X)=0$;

- $C$ is connected;

- $f$ satisfies (usc), (Bdr-s), and (Conn).

Then $\operatorname{Iter}(f, C, X) \geq 5$. 
The optimality of these estimates follows from the optimality of the corresponding estimates for functions.

Remark 2.13 Example 4.9 shows that statement (3) is the best one can expect under assumption (Bdr-w) (note that we assumed (Bdr-s) in statement (4)). In that example indeed $X$ is $\mathbb{R}^{2}, C$ is a contractible compact set which satisfies the fixed point property for functions, and all images of $f$ are convex sets.

\section{Proofs}

\subsection{Topological lemmata}

The five lemmata we collect in this section are the technical core of this paper.

The first one is standard point-set topology. The statements may seem trivial: nevertheless, at least (1), (21), and (3) are false without local connectedness assumptions.

Lemma 3.1 Let $Y$ be a locally connected topological space.

Then the following implications are true.

(1) If $V \subseteq Y$ is any subset, and $V^{\prime}$ is a connected component of $V$, then $\partial V^{\prime} \subseteq \partial V$.

(2) If $V \subseteq Y$ is closed, and $V^{\prime}$ is a connected component of $V$, then $\partial V^{\prime}=V^{\prime} \cap \partial V$.

(3) For every family $\left\{A_{i}\right\}_{i \in I}$ of subsets of $Y$ we have that

$$
\partial\left(\bigcup_{i \in I} A_{i}\right) \subseteq \operatorname{Clos}\left(\bigcup_{i \in I} \partial A_{i}\right) .
$$

(4) Let us assume that $Y$ is connected, $A \subseteq Y$ is an open subset such that $Y \backslash A$ is connected, and $A^{\prime}$ is a connected component of $A$. Then $Y \backslash A^{\prime}$ is connected.

Proof.

Statement (1). Let $x \in \partial V^{\prime}$. Then $x \in \operatorname{Clos}\left(V^{\prime}\right) \subseteq \operatorname{Clos}(V)$, hence either $x \in \partial V$ or $x \in \operatorname{Int}(V)$. Assume by contradiction that $x \in \operatorname{Int}(V)$. Since $Y$ is locally connected there exists a connected neighborhood $U$ of $x$ contained in $V$. Since $U$ is connected it is necessarily contained in $V^{\prime}$, but this implies that $x \in \operatorname{Int}\left(V^{\prime}\right)$ and contradicts the assumption that $x \in \partial V^{\prime}$.

Statement (2). We have that $\partial V^{\prime} \subseteq V^{\prime}$ because $V^{\prime}$ is closed, and $\partial V^{\prime} \subseteq \partial V$ because of the statement (11). The opposite inclusion $V^{\prime} \cap \partial V \subseteq \partial V^{\prime}$ is trivial (it holds true also without the local connectedness of $Y$ or the closedness of $V$ ). 
Statement (3). Let $x$ be a point in the boundary of the union, and let $U$ be any connected neighborhood of $x$. By assumption there exists $i_{0} \in I$ such that $U \cap A_{i_{0}} \neq \emptyset$ and $U \backslash A_{i_{0}} \neq \emptyset$. By the connectedness of $U$ this implies that $U \cap \partial A_{i_{0}} \neq \emptyset$. Since $x$ has a fundamental system of connected neighborhoods, this is enough to conclude that $x$ belongs to the closure of the union of the boundaries.

Statement (4). If $A$ is connected the conclusion is trivial. Otherwise, let $\left\{A_{i}\right\}_{i \in I}$ be the set of connected components of $A \backslash A^{\prime}$ so that

$$
Y \backslash A^{\prime}=(Y \backslash A) \cup \bigcup_{i \in I} A_{i}=\bigcup_{i \in I}\left[(Y \backslash A) \cup A_{i}\right]
$$

Thus it is enough to show that $(Y \backslash A) \cup A_{i}$ is connected for every $i \in I$. Since $A_{i}$ is a nontrivial subset of the connected space $Y$, we have that $\partial A_{i} \neq \emptyset$, hence by statement (1)

$$
\emptyset \neq \partial A_{i} \subseteq \partial A=\partial(Y \backslash A) \subseteq Y \backslash A .
$$

Since $\operatorname{Clos}\left(A_{i}\right)$ is also connected it follows that $(Y \backslash A) \cup A_{i}=(Y \backslash A) \cup \operatorname{Clos}\left(A_{i}\right)$ is the union of two connected sets with nonempty intersection, hence it is connected.

The second lemma relates the cohomological assumption on the space to the connectedness of the boundary of suitable subsets. We use this result every time we want to prove that the boundary of an open set is connected.

Lemma 3.2 Let $Y$ be a paracompact Hausdorff topological space such that $\check{H}^{1}(Y)=0$. Let $A \subseteq Y$ be a connected open set such that $Y \backslash A$ is also connected.

Then $\partial A$ is connected.

PROOF. We recall that a topological space is connected if and only if its 0-dimensional reduced Alexander cohomology group (with any coefficient group) is trivial.

Let us consider the long exact sequence of reduced Alexander cohomology groups for the pair $(Y, \operatorname{Clos}(A))$ (see [3, Theorem 2.13]):

$$
\ldots \longrightarrow \widetilde{H}^{0}(\operatorname{Clos}(A)) \longrightarrow \widetilde{H}^{1}(Y, \operatorname{Clos}(A)) \longrightarrow \widetilde{H}^{1}(Y) \longrightarrow \ldots
$$

In this sequence we have that $\widetilde{H}^{0}(\operatorname{Clos}(A))=0$ because $\operatorname{Clos}(A)$ is connected, and $\widetilde{H}^{1}(Y)=0$. This implies that $\widetilde{H}^{1}(Y, \operatorname{Clos}(A))=0$.

By the strong excision property in paracompact Hausdorff spaces (see Exercise $6 \mathrm{~B}$ in [3, p. 89] or Theorem 5 in [11, p. 318]) we can subtract $A$ to both $Y$ and $\operatorname{Clos}(A)$ obtaining that

$$
\widetilde{H}^{1}(Y \backslash A, \partial A)=\widetilde{H}^{1}(Y, \operatorname{Clos}(A))=0 .
$$

Thus in the long exact sequence for the pair $(Y \backslash A, \partial A)$

$$
\ldots \longrightarrow \widetilde{H}^{0}(Y \backslash A) \longrightarrow \widetilde{H}^{0}(\partial A) \longrightarrow \widetilde{H}^{1}(Y \backslash A, \partial A) \longrightarrow \ldots
$$


we have that $\widetilde{H}^{1}(Y \backslash A, \partial A)=0$ and $\widetilde{H}^{0}(Y \backslash A)=0$ because $Y \backslash A$ is connected. It follows that $\widetilde{H}^{0}(\partial A)=0$, which is equivalent to say that $\partial A$ is connected.

The following result is used in the sequel every time we prove the existence of a fifth iteration. We state and prove it under the joint hypotheses of Lemma 3.1 and Lemma 3.2. We suspect it can be true also without the local connectedness assumption, but in that case the proof could be much more involved. On the contrary, the cohomological assumption is likely to be necessary.

Lemma 3.3 Let $Y$ be a paracompact Hausdorff locally connected topological space such that $\check{H}^{1}(Y)=0$. Let $K_{1}, K_{2}, U$ be three subsets such that

(i) $K_{1} \cap K_{2}=\emptyset$;

(ii) $U$ is open and $Y \backslash U$ is connected;

(iii) $\partial U \subseteq K_{1} \cup K_{2}$;

(iv) $\partial U \cap K_{1}$ and $\partial U \cap K_{2}$ are closed sets.

Then $U$ is the disjoint union of two subsets $U_{1}$ and $U_{2}$ such that $\partial U_{1} \subseteq \operatorname{Clos}\left(K_{1}\right)$ and $\partial U_{2} \subseteq \operatorname{Clos}\left(K_{2}\right)$.

Proof. Let $U^{\prime}$ be any connected component of $U$. By (ii) and statement (41) of Lemma 3.1 we have that $Y \backslash U^{\prime}$ is connected, and therefore from Lemma 3.2 we deduce that $\partial U^{\prime}$ is connected. Due to statement (11) of Lemma 3.1 and assumption (iii) we have that $\partial U^{\prime} \subseteq \partial U \subseteq K_{1} \cup K_{2}$. We can therefore write

$$
\partial U^{\prime}=\left(\partial U^{\prime} \cap K_{1}\right) \cup\left(\partial U^{\prime} \cap K_{2}\right) .
$$

By assumptions (i) and (iv), the two terms in the right hand side are closed and disjoint, hence one of them must be empty. This proves that every connected component $U^{\prime}$ of $U$ satisfies either $\partial U^{\prime} \subseteq K_{1}$ or $\partial U^{\prime} \subseteq K_{2}$.

Let $\left\{U_{i}\right\}_{i \in I}$ be the set of connected components of $U$ whose boundary is contained in $K_{1}$, and let $\left\{U_{j}\right\}_{j \in J}$ be the set of connected components of $U$ whose boundary is contained in $K_{2}$. Let us set

$$
U_{1}:=\bigcup_{i \in I} U_{i}, \quad U_{2}:=\bigcup_{j \in J} U_{j} .
$$

It is clear that $U_{1} \cap U_{2}=\emptyset$ and $U_{1} \cup U_{2}=U$. Moreover from statement (3) of Lemma 3.1 we have that

$$
\partial U_{1}=\partial\left(\bigcup_{i \in I} U_{i}\right) \subseteq \operatorname{Clos}\left(\bigcup_{i \in I} \partial U_{i}\right) \subseteq \operatorname{Clos}\left(K_{1}\right)
$$


and similarly for $U_{2}$.

A first consequence of Lemma 3.3 is the following result, which is the main tool in the proof of Theorem 2.8 .

Lemma 3.4 It is not possible to decompose the unit square $[0,1] \times[0,1]$ as the disjoint union of subsets $\mathcal{A}_{i}(i=0,1,2,3,4)$ satisfying the following properties:

(A1) $(0,1) \in \mathcal{A}_{2}$ and $(1,1) \in \mathcal{A}_{1}$;

(A2) $\mathcal{A}_{4}$ does not intersect the side $[0,1] \times\{1\}$;

(A3) $\mathcal{A}_{0}$ does not intersect the other three sides;

(A4) $\mathcal{A}_{3}, \mathcal{A}_{2} \cup \mathcal{A}_{4}, \mathcal{A}_{1} \cup \mathcal{A}_{3} \cup \mathcal{A}_{4}$ are closed sets;

(A5) $\mathcal{A}_{0}$ is an open set and $\partial \mathcal{A}_{0} \subseteq \mathcal{A}_{2} \cup \mathcal{A}_{3} \cup \mathcal{A}_{4}$.

Proof. Let us set for simplicity $Q:=[0,1] \times[0,1]$. First of all we show that, up to modifying the sets $\mathcal{A}_{0}, \ldots, \mathcal{A}_{4}$, we can assume that they fulfil (A1) through (A 5 ) and also the following additional property:

(A6) $Q \backslash \mathcal{A}_{0}$ is connected.

Let indeed $P$ be the union of the three sides considered in $(\mathrm{A} 3)$. By $(\mathrm{A} 3)$ the closed set $Q \backslash \mathcal{A}_{0}$ contains the connected set $P$. Let $\mathcal{V}$ be the connected component of $Q \backslash \mathcal{A}_{0}$ containing $P$. Let us set $\widetilde{\mathcal{A}}_{0}:=Q \backslash \mathcal{V}$ and $\widetilde{\mathcal{A}}_{i}:=\mathcal{A}_{i} \cap \mathcal{V}$ for $i=1,2,3,4$.

It is easy to see that the sets $\widetilde{\mathcal{A}}_{0}, \ldots, \widetilde{\mathcal{A}}_{4}$ are disjoint and satisfy assumptions (A1) through (A 4 ), and (A6). Moreover $\widetilde{\mathcal{A}}_{0}$ is open because $\mathcal{V}$ is closed. Finally, from statement (2) of Lemma 3.1 we have that

$$
\partial \widetilde{\mathcal{A}}_{0}=\partial \mathcal{V}=\partial\left(Q \backslash \mathcal{A}_{0}\right) \cap \mathcal{V}=\partial \mathcal{A}_{0} \cap \mathcal{V} \subseteq\left(\mathcal{A}_{2} \cup \mathcal{A}_{3} \cup \mathcal{A}_{4}\right) \cap \mathcal{V}=\widetilde{\mathcal{A}}_{2} \cup \widetilde{\mathcal{A}}_{3} \cup \widetilde{\mathcal{A}}_{4},
$$

which proves also (A 5 ).

Roughly speaking, what we have done in this first part of the proof is to fill the holes of $\mathcal{A}_{0}$ which do not touch $P$, as shown in the following picture ( $P$ is the union of the lower and lateral sides of the squares).
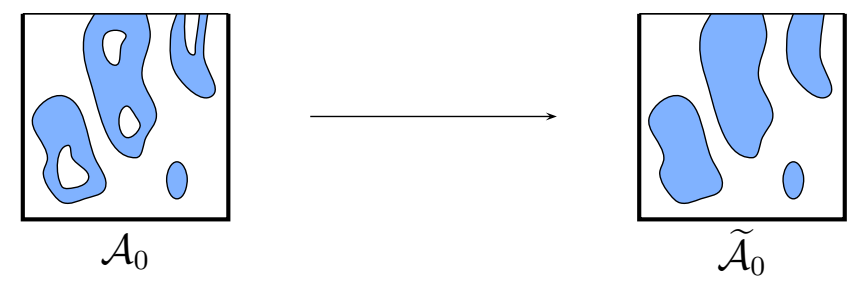
From now on we drop tildes and we assume that $\mathcal{A}_{0}, \ldots, \mathcal{A}_{4}$ satisfy (A1) through (A6).

Since of course $\check{H}^{1}(Q)=0$, we can apply Lemma 3.3 with $Y=Q, K_{1}=\mathcal{A}_{3}$, $K_{2}=\mathcal{A}_{2} \cup \mathcal{A}_{4}, U=\mathcal{A}_{0}$. We obtain that $\mathcal{A}_{0}$ is the disjoint union of two sets $\mathcal{A}_{0}^{\prime}$ and $\mathcal{A}_{0}^{\prime \prime}$ such that $\partial \mathcal{A}_{0}^{\prime} \subseteq \mathcal{A}_{3}$ and $\partial \mathcal{A}_{0}^{\prime \prime} \subseteq \mathcal{A}_{2} \cup \mathcal{A}_{4}$. Together with (A4) this implies in particular that $\mathcal{A}_{0}^{\prime} \cup \mathcal{A}_{1} \cup \mathcal{A}_{3} \cup \mathcal{A}_{4}$ and $\mathcal{A}_{0}^{\prime \prime} \cup \mathcal{A}_{2} \cup \mathcal{A}_{4}$ are closed subsets of $Q$.

Let us consider now the side $S:=[0,1] \times\{1\}$, which can be written in the form

$$
S=\left[S \cap\left(\mathcal{A}_{0}^{\prime} \cup \mathcal{A}_{1} \cup \mathcal{A}_{3} \cup \mathcal{A}_{4}\right)\right] \cup\left[S \cap\left(\mathcal{A}_{0}^{\prime \prime} \cup \mathcal{A}_{2} \cup \mathcal{A}_{4}\right)\right]=: S_{1} \cup S_{2} .
$$

By (A2) we have that $S \cap \mathcal{A}_{4}=\emptyset$, which proves that $S_{1} \cap S_{2}=\emptyset$. By (A1) we have that $(1,1) \in S_{1}$ and $(0,1) \in S_{2}$. Since $S_{1}$ and $S_{2}$ are closed sets, this contradicts the connectedness of $S$.

The last lemma is the set-valued extension of a well known result for continuous functions.

Lemma 3.5 Let $X$ be a topological space, let $C \subseteq X$ be a closed subset, and let $f$ : $C \rightarrow \mathcal{P}_{\star}(X)$. Given $A \subseteq C$, let $f(A)$ be the image of $A$, defined as the union of $f(x)$ when $x$ ranges in $A$.

If $f$ satisfies (usc) and (Conn), and $A$ is connected, then $f(A)$ is connected.

Proof. We argue by contradiction. Let us assume that $U$ and $V$ are open subsets of $X$ such that $f(A) \cap U$ and $f(A) \cap V$ are nonempty disjoint sets whose union is $f(A)$. Let $x \in A$. Since $f(x)$ is connected and contained in $f(A)$, it is clear that either $f(x) \subseteq U$ or $f(x) \subseteq V$. Therefore if we now define

$$
U_{1}:=\{x \in A: f(x) \subseteq U\}, \quad V_{1}:=\{x \in A: f(x) \subseteq V\},
$$

we have found two nonempty disjoint open subsets of $A$ whose union is $A$. This contradicts the connectedness of $A$.

\subsection{Proof of Proposition 2.5}

As a general fact we recall that, since each $C_{i}$ is a closed set, the $\operatorname{closure} \operatorname{Clos}_{i}(Z)$ in $C_{i}$ of any subset $Z \subseteq C_{i}$ coincides with the closure $\operatorname{Clos}(Z)$ of $Z$ in $X$.

Statement (1) This can be easily proved by induction using the definition of $C_{n}$ and the continuity of $f$. 
Statement (2) Since $C_{n+2}$ is closed we have that $f\left(\partial_{n+1} C_{n+2}\right) \subseteq f\left(C_{n+2}\right) \subseteq C_{n+1}$. Moreover

$$
\partial_{n+1} C_{n+2}=\partial_{n+1}\left(C_{n+1} \backslash C_{n+2}\right)=\partial_{n+1} A_{n+1} \subseteq \operatorname{Clos}_{n+1}\left(A_{n+1}\right)=\operatorname{Clos}\left(A_{n+1}\right),
$$

hence

$$
f\left(\partial_{n+1} C_{n+2}\right) \subseteq f\left(\operatorname{Clos}\left(A_{n+1}\right)\right) \subseteq \operatorname{Clos}\left(f\left(A_{n+1}\right)\right) \subseteq \operatorname{Clos}\left(A_{n}\right)=\operatorname{Clos}_{n}\left(C_{n} \backslash C_{n+1}\right) .
$$

We have thus established that $f\left(\partial_{n+1} C_{n+2}\right) \subseteq C_{n+1} \cap \operatorname{Clos}_{n}\left(C_{n} \backslash C_{n+1}\right)$, which is equivalent to say that $f\left(\partial_{n+1} C_{n+2}\right) \subseteq \partial_{n} C_{n+1}$.

Statement (3) Let us argue by induction. The case $n=0$ follows from the assumption that $f(\partial C) \subseteq C$. Assume now that $\partial_{n} C_{n+1} \subseteq C_{n+2}$ for some given $n$. By statement (2) and the inductive hypothesis we have that $f\left(\partial_{n+1} C_{n+2}\right) \subseteq \partial_{n} C_{n+1} \subseteq C_{n+2}$, which proves that $\partial_{n+1} C_{n+2} \subseteq C_{n+3}$ and completes the induction.

Statement (4) By statement (3) we have that

$$
\operatorname{Clos}\left(A_{n}\right)=\operatorname{Clos}_{n}\left(A_{n}\right)=A_{n} \cup \partial_{n} A_{n}=A_{n} \cup \partial_{n}\left(C_{n} \backslash A_{n}\right)=A_{n} \cup \partial_{n} C_{n+1} \subseteq A_{n} \cup C_{n+2},
$$

hence, since $C_{n+2}$ is closed, $\operatorname{Clos}\left(A_{n} \cup C_{n+2}\right)=\operatorname{Clos}\left(A_{n}\right) \cup \operatorname{Clos}\left(C_{n+2}\right) \subseteq A_{n} \cup C_{n+2}$, which completes the proof.

\subsection{Proof of Theorem 2.7}

Statement (1) Trivial because $\partial C \subseteq C_{2}$.

Statement (2) If $C=X$, then $\operatorname{Iter}(f, C, X)=+\infty$. If $C$ is a proper subset of the connected space $X$, then $\partial C \neq \emptyset$, which proves that $\operatorname{Iter}(f, C, X) \geq 2$. Assume by contradiction that it is exactly 2. This means that $X=A_{0} \cup A_{1} \cup A_{2}$. Applying statement (4) of Proposition 2.5 with $n=0$ and $n=1$, we deduce that both $A_{0} \cup A_{2}$ and $A_{1}$ are nonempty closed sets and this contradicts the connectedness of $X$.

Statement (3) By the previous statement we know that $\operatorname{Iter}(f, C, X) \geq 3$. Assume by contradiction that it is exactly 3. This means that $C=A_{1} \cup A_{2} \cup A_{3}$. Applying statement (4) of Proposition 2.5 with $n=1$ and $n=2$, we deduce that both $A_{1} \cup A_{3}$ and $A_{2}$ are nonempty closed sets and this contradicts the connectedness of $C$. 
Statement (4) Since $X$ and $C$ are connected, from statement (3) we know that Iter $(f, C, X) \geq 4$. Assume now by contradiction that it is exactly 4. Applying statement (4) of Proposition 2.5 with $n=1,2,3$ we have that $A_{1} \cup A_{3} \cup A_{4}, A_{2} \cup A_{4}$, and $A_{3}$ are nonempty closed subsets of $X$.

Since $\partial A_{0}=\partial C \subseteq A_{2} \cup A_{3} \cup A_{4}$, we can apply Lemma 3.3 with $Y=X, K_{1}=A_{3}$, $K_{2}=A_{2} \cup A_{4}, U=A_{0}$. We obtain that $A_{0}$ is the disjoint union of two sets $A_{0}^{\prime}$ and $A_{0}^{\prime \prime}$ such that $\partial A_{0}^{\prime} \subseteq A_{3}$ and $\partial A_{0}^{\prime \prime} \subseteq A_{2} \cup A_{4}$. This implies in particular that $A_{0}^{\prime} \cup A_{1} \cup A_{3} \cup A_{4}$ and $A_{0}^{\prime \prime} \cup A_{2} \cup A_{4}$ are closed subsets of $X$.

Let us consider now the connected set $f(C)$, and let us write

$$
f(C)=\left[f(C) \cap\left(A_{0}^{\prime} \cup A_{1} \cup A_{3} \cup A_{4}\right)\right] \cup\left[f(C) \cap\left(A_{0}^{\prime \prime} \cup A_{2} \cup A_{4}\right)\right]=: F_{1} \cup F_{2} .
$$

Then $F_{1}$ and $F_{2}$ are closed subsets of $f(C)$. They are also nonempty because $f(C)$ intersects $A_{1}, A_{2}$ and $A_{3}$. Finally, they are disjoint because $f(C) \cap A_{4}=\emptyset$. This contradicts the connectedness of $f(C)$.

Statement (5) Let $r: X \backslash \operatorname{Int}(C) \rightarrow \partial C$ be a retraction, and let

$$
g(x):= \begin{cases}f(x) & \text { if } f(x) \in C \\ r(f(x)) & \text { if } f(x) \notin \operatorname{Int}(C) .\end{cases}
$$

It is not difficult to see that $g: C \rightarrow C$ is continuous (one only needs to verify that it is well defined when $f(x) \in \partial C$ ). Since $C$ satisfies the fixed point property there exists $x_{0} \in C$ such that $g\left(x_{0}\right)=x_{0}$. We claim that $x_{0}$ is indeed a fixed point of $f$.

If $f\left(x_{0}\right) \in C$ then $x_{0}=g\left(x_{0}\right)=f\left(x_{0}\right)$ and so $x_{0}$ is also a fixed point of $f$. Assume now by contradiction that $f\left(x_{0}\right) \notin C$. Since $f(\partial C) \subseteq C$, this implies that $x_{0} \notin \partial C$. On the other hand, in this case $g\left(x_{0}\right)=r\left(f\left(x_{0}\right)\right) \in \partial C$, which is absurd. This completes the proof.

\subsection{Proof of Theorem 2.8}

Let $C^{\prime}$ be a connected component of $C$, and let $X^{\prime}$ be the connected component of $X$ containing $C^{\prime}$. Since $f$ is homotopic to the identity it is easy to see that $f$ maps $C^{\prime}$ to $X^{\prime}$. From now on we can therefore assume that $C$ and $X$ are connected, so that by statement (3) of Theorem 2.7 we have that $\operatorname{Iter}(f, C, X) \geq 4$.

Assume now that it is exactly 4. Applying statement (4) of Proposition 2.5 with $n=1,2,3$ we have that $A_{1} \cup A_{3} \cup A_{4}, A_{2} \cup A_{4}$, and $A_{3}$ are nonempty closed subsets of $X$. Moreover $A_{0}$ is open and $\partial A_{0} \subseteq A_{2} \cup A_{3} \cup A_{4}$.

Step 1. We prove that $\partial C \cap A_{3} \neq \emptyset$. 
Let us assume indeed by contradiction that $\partial C=\partial A_{0} \subseteq A_{2} \cup A_{4}$, hence in particular that $A_{0} \cup A_{2} \cup A_{4}$ is a closed set. Now we consider the connected set $f(C)$ and we write

$$
f(C)=\left[f(C) \cap\left(A_{1} \cup A_{3} \cup A_{4}\right)\right] \cup\left[f(C) \cap\left(A_{0} \cup A_{2} \cup A_{4}\right)\right]=: F_{1} \cup F_{2} .
$$

Then $F_{1}$ and $F_{2}$ are closed subsets of $f(C)$. They are also nonempty because $f(C)$ intersects $A_{1}, A_{2}$ and $A_{3}$. Finally, they are disjoint because $f(C) \cap A_{4}=\emptyset$. This contradicts the connectedness of $f(C)$.

Step 2. Let $x_{0} \in \partial C \cap A_{3}$. We show that there exists a continuous curve $\gamma:[0,1] \rightarrow C$ such that $\gamma(0)=x_{0}$ and $\gamma(1) \in \partial C \cap A_{2}$.

To begin with, let us consider the curve $\gamma_{1}:[0,1] \rightarrow C$ defined by $\gamma_{1}(t)=\Phi\left(x_{0}, t\right)$. This curve takes its values in $C$ because the homotopy sends $\partial C$ back to $C$. We can therefore extend it to a curve $\gamma_{2}:[0,2] \rightarrow X$ by setting

$$
\gamma_{2}(t):= \begin{cases}\gamma_{1}(t) & \text { if } t \in[0,1] \\ f\left(\gamma_{1}(t-1)\right) & \text { if } t \in[1,2]\end{cases}
$$

The curve $\gamma_{2}$ is continuous (one only needs to check that it is well defined for $t=1$ ). Moreover $\gamma_{2}(1)=f\left(x_{0}\right) \in A_{2}, \gamma_{2}(2)=f\left(f\left(x_{0}\right)\right) \in A_{1}$, and for every $t \in[1,2]$ we have that $\gamma_{2}(t) \in f(C) \subseteq A_{0} \cup A_{1} \cup A_{2} \cup A_{3}$.

We claim that $\gamma_{2}(t) \in A_{0}$ for some $t \in[1,2]$. Assume indeed that $\gamma_{2}(t) \in A_{1} \cup A_{2} \cup A_{3}$ for every $t \in[1,2]$. Then

$$
[1,2]=\left\{t \in[1,2]: \gamma_{1}(t) \in A_{2} \cup A_{4}\right\} \cup\left\{t \in[1,2]: \gamma_{2}(t) \in A_{1} \cup A_{3} \cup A_{4}\right\}=: I_{1} \cup I_{2} .
$$

Thus $I_{1}$ and $I_{2}$ are closed sets, and they are nonempty because $1 \in I_{1}$ and $2 \in$ $I_{2}$. Moreover they are disjoint because $\gamma_{2}([1,2]) \cap A_{4}=\emptyset$, and this contradicts the connectedness of $[1,2]$.

Let us set now $t_{\star}:=\inf \left\{t \in[1,2]: \gamma_{2}(t) \in A_{0}\right\}$. From the definition of infimum it is clear that $\gamma_{2}(t) \in C$ for every $t \in\left[0, t_{\star}\right]$ and $\gamma_{2}\left(t_{\star}\right) \in \partial A_{0}=\partial C$. We claim that $\gamma_{2}\left(t_{\star}\right) \in A_{2}$. Let us consider indeed

$$
\left[1, t_{\star}\right]=\left\{t \in\left[1, t_{\star}\right]: \gamma_{2}(t) \in A_{1} \cup A_{3}\right\} \cup\left\{t \in\left[1, t_{\star}\right]: \gamma_{2}(t) \in A_{2}\right\} .
$$

Once again the two sets in the right hand side are closed and disjoint, and the second one is nonempty because it contains $t=1$. By the connectedness of $\left[1, t_{\star}\right]$ it follows that the first one is empty and therefore $\gamma_{2}\left(t_{\star}\right) \in A_{2}$.

The curve $\gamma$ we are looking for is just (a reparametrization of) the restriction of $\gamma_{2}$ to the interval $\left[0, t_{\star}\right]$.

Step 3. Let $\gamma$ be the curve of step 2, and let

$$
\mathcal{A}_{i}:=\left\{(\tau, t) \in[0,1] \times[0,1]: \Phi(\gamma(\tau), t) \in A_{i}\right\}
$$


for $i=0,1,2,3,4$. If we show that the $\mathcal{A}_{i}$ 's satisfy assumptions (A1) through (A 5 ) of Lemma 3.4 we have a contradiction.

Since $\gamma(0) \in A_{3}$ we have that $\Phi(\gamma(0), 1)=f(\gamma(0)) \in A_{2}$, hence $(0,1) \in \mathcal{A}_{2}$. Since $\gamma(1) \in A_{2}$ we have that $\Phi(\gamma(1), 1)=f(\gamma(1)) \in A_{1}$, hence $(1,1) \in \mathcal{A}_{1}$. This proves (A1).

Since the image of $f$ is contained in $A_{0} \cup A_{1} \cup A_{2} \cup A_{3}$ it follows that $\Phi(\gamma(\tau), 1)=$ $f(\gamma(\tau)) \notin A_{4}$ for every $\tau \in[0,1]$, which proves (A[2).

Since $\gamma(0)$ and $\gamma(1)$ belong to $\partial C$, and $\Phi$ sends $\partial C$ back to $C$, we have that $\Phi(\gamma(0), t)$ and $\Phi(\gamma(1), t)$ are in $C$ for every $t \in[0,1]$. Since also $\Phi(\gamma(\tau), 0)=\gamma(\tau) \in C$ for every $\tau \in[0,1]$, this proves $(\mathrm{A}[3)$.

Finally, (A44) and (A5) follow from the continuity of $\Phi(\gamma(\tau), t)$ and the analogous properties of the $A_{i}$ 's.

\subsection{Proof of Proposition 2.11}

Statement (1) This can be easily proved by induction using the definition of $C_{n}$ and the upper semicontinuity of $f$.

Statement (2) The set $A_{0}=X \backslash C$ is open because $C$ is closed. Now since

$$
A_{1}=\{x \in C: f(x) \cap C=\emptyset\}=\left\{x \in C: f(x) \subseteq A_{0}\right\},
$$

and since $f$ satisfies (usc), we have that $A_{1}$ is an open subset of $C$. In order to conclude that it is also an open subset of $X$ it suffices to prove that $A_{1} \cap \partial C=\emptyset$. This follows from (Bdr-w).

Statement (3) Let $x \in \partial_{1} C_{2}$. Since $C_{2}$ is closed we have that $x \in C_{2}$, hence either $x \in C_{3}$ or $x \in A_{2}$. Let us assume by contradiction that $x \in A_{2}$. Then $f(x) \subseteq A_{0} \cup A_{1}$ and $f(x) \cap A_{1} \neq \emptyset$. Since $A_{0}$ and $A_{1}$ are open sets, and $f(x)$ is connected, we have that $f(x) \subseteq A_{1}$. This means that actually $A_{2}=\left\{x \in C_{1}: f(x) \subseteq A_{1}\right\}$, and thus it is an open subset of $C_{1}$ contained in $C_{2}$. Therefore if $x \in A_{2}$ then $x \in \operatorname{Int}_{1}\left(C_{2}\right)$, which contradicts the initial assumption that $x \in \partial_{1} C_{2}$.

Statement (4) The argument is the same used in the proof of statement (4) of Proposition 2.5. Since $C_{1}$ is closed and $\partial_{1} C_{2} \subseteq C_{3}$ we have that

$$
\operatorname{Clos}\left(A_{1}\right)=\operatorname{Clos}_{1}\left(A_{1}\right)=A_{1} \cup \partial_{1} A_{1}=A_{1} \cup \partial_{1}\left(C_{1} \backslash A_{1}\right)=A_{1} \cup \partial_{1} C_{2} \subseteq A_{1} \cup C_{3},
$$

hence, since $C_{3}$ is closed, $\operatorname{Clos}\left(A_{1} \cup C_{3}\right)=\operatorname{Clos}\left(A_{1}\right) \cup \operatorname{Clos}\left(C_{3}\right)=A_{1} \cup C_{3}$. 
Statement (5) We argue more or less as in the proof of statement (3).

Let $x \in \partial_{2} C_{3}$. Since $C_{3}$ is closed we have that $x \in C_{3}$, hence either $x \in C_{4}$ or $x \in A_{3}$. Let us assume by contradiction that $x \in A_{3}$. Then $f(x) \subseteq\left(A_{0} \cup A_{2}\right) \cup A_{1}$ and $f(x) \cap A_{2} \neq \emptyset$. Since $A_{0} \cup A_{2}$ and $A_{1}$ are open sets, and $f(x)$ is connected, we have that $f(x) \subseteq A_{0} \cup A_{2}$. This means that actually $A_{3}=\left\{x \in C_{2}: f(x) \subseteq A_{0} \cup A_{2}\right\}$, and thus it is an open subset of $C_{2}$ contained in $C_{3}$. Therefore if $x \in A_{3}$ then $x \in \operatorname{Int}_{2}\left(C_{3}\right)$, which contradicts the initial assumption that $x \in \partial_{2} C_{3}$.

Statement (6) Same proof of statement (4) with indices increased by 1.

Statement (7) Let $x \in \partial_{3} C_{4} \cap A_{4}$. Since $x \in A_{4}$ we know that $f(x) \cap A_{3} \neq \emptyset$ and $f(x) \subseteq A_{0} \cup A_{1} \cup A_{2} \cup A_{3}$. Let us assume by contradiction that $f(x) \cap A_{2}=\emptyset$, hence that $f(x)$ is contained in the open set $A_{0} \cup A_{1} \cup A_{3}$. Now consider $U:=\left\{x \in C_{3}\right.$ : $\left.f(x) \subseteq A_{0} \cup A_{1} \cup A_{3}\right\}$. It is an open subset of $C_{3}$ which is contained in $C_{4}$ (all points in $U$ lie indeed in $\left.C_{4}\right)$. Since $x \in U$, we conclude that $x \in \operatorname{Int}_{3}\left(C_{4}\right)$, which contradicts the initial assumption that $x \in \partial_{3} C_{4}$.

\subsection{Proof of Theorem 2.12}

Statement (1) Trivial because $\partial C \subseteq C_{2}$.

Statement (2) If $C=X$, then Iter $(f, C, X)=+\infty$. If $C$ is a proper subset of the connected space $X$, then $\partial C \neq \emptyset$, which proves that $\operatorname{Iter}(f, C, X) \geq 2$. Assume by contradiction that it is exactly 2. This means that $X=A_{0} \cup A_{1} \cup A_{2}$. By statements (2) and (4) of Proposition 2.11 we know that both $A_{0} \cup A_{2}$ and $A_{1}$ are nonempty open sets and this contradicts the connectedness of $X$.

Statement (3) By the previous statement we know that $\operatorname{Iter}(f, C, X) \geq 3$. Assume by contradiction that it is exactly 3 . This means that $C=A_{1} \cup A_{2} \cup A_{3}$. By statements (4) and (6) of Proposition 2.11 we know that in this case both $A_{1} \cup A_{3}$ and $A_{2}$ are nonempty closed sets and this contradicts the connectedness of $C$.

Statement (4) Since $X$ and $C$ are connected, from statement (3) we know that $\operatorname{Iter}(f, C, X) \geq 4$. Assume now by contradiction that it is exactly 4 . From statements (11), (44), and (6) of Proposition 2.11 we know that $A_{1} \cup A_{3} \cup A_{4}, A_{2} \cup A_{4}$ and $A_{3} \cup A_{4}=C_{3}$ are closed sets, but we don't know whether $A_{3}$ is closed or not.

Let us prove that in any case $A_{3} \cap \partial C$ is closed. Indeed, since

$$
\operatorname{Clos}\left(A_{3}\right)=\operatorname{Clos}_{3}\left(A_{3}\right)=A_{3} \cup \partial_{3} A_{3}=A_{3} \cup \partial_{3}\left(C_{3} \backslash A_{3}\right)=A_{3} \cup \partial_{3} C_{4},
$$


and since $\partial_{3} C_{4} \subseteq C_{4}=A_{4}$, we have that $A_{3} \cap \partial C$ is closed if and only if $\partial_{3} C_{4} \cap \partial C=\emptyset$. Let us assume by contradiction that there exists $x \in \partial_{3} C_{4} \cap \partial C$. By (Bdr-s) we have that $f(x) \subseteq C$, hence $f(x) \cap A_{0}=\emptyset$ and therefore

$$
f(x)=\left[f(x) \cap\left(A_{1} \cup A_{3} \cup A_{4}\right)\right] \cup\left[f(x) \cap\left(A_{2} \cup A_{4}\right)\right]=: F_{1} \cup F_{2} .
$$

Thus $F_{1}$ and $F_{2}$ are closed subsets of $f(x)$. Moreover, since $x \in \partial_{3} C_{4}=\partial_{3} C_{4} \cap A_{4}$, from statement (7) of Proposition 2.11 we deduce that $F_{1}$ and $F_{2}$ are nonempty. Finally, they are disjoint because $f(x) \cap A_{4}=\emptyset$. This contradicts the connectedness of $f(x)$.

Once we know that $A_{3} \cap \partial C$ is closed we can proceed as in the case of functions. We apply Lemma 3.3 with $Y=X, K_{1}=A_{3}, K_{2}=A_{2} \cup A_{4}, U=A_{0}$ and we obtain that $A_{0}$ is the disjoint union of two sets $A_{0}^{\prime}$ and $A_{0}^{\prime \prime}$ such that $\partial A_{0}^{\prime} \subseteq \operatorname{Clos}\left(A_{3}\right) \subseteq A_{3} \cup A_{4}$ and $\partial A_{0}^{\prime \prime} \subseteq \operatorname{Clos}\left(A_{2} \cup A_{4}\right)=A_{2} \cup A_{4}$. This implies in particular that $A_{0}^{\prime} \cup A_{1} \cup A_{3} \cup A_{4}$ and $A_{0}^{\prime \prime} \cup A_{2} \cup A_{4}$ are closed subsets of $X$.

Now we consider $f(C)$, which is a connected set because of Lemma 3.5, and we write

$$
f(C)=\left[f(C) \cap\left(A_{0}^{\prime} \cup A_{1} \cup A_{3} \cup A_{4}\right)\right] \cup\left[f(C) \cap\left(A_{0}^{\prime \prime} \cup A_{2} \cup A_{4}\right)\right] .
$$

Since $f(C) \cap A_{4}=\emptyset$, the two sets in brackets in the right hand side are disjoint. They are also nonempty because $f(C)$ intersects $A_{1}, A_{2}$ and $A_{3}$. Finally, they are closed subsets of $f(C)$.

This contradicts the connectedness of $f(C)$.

\section{Examples}

The first four examples show that the estimates of $\operatorname{Iter}(f, C, X)$ given in the first four statements of Theorem 2.7 are optimal.

Example 4.1 Let $X:=\{0\} \cup[2,4]$ with the topology inherited as a subset of the real line, let $C:=\{0,4\}$, and let $f: C \rightarrow X$ be defined by $f(0)=3$ and $f(4)=0$.

Then $X, C$, and $f$ satisfy the assumptions of Problem 2.1 (in this case indeed $\partial C=\{4\})$, and $\operatorname{Iter}(f, C, X)=2$.

Example 4.2 Let $X:=\mathbb{R}$ with the usual topology, let $C:=\{0\} \cup[2,4]$, and let $f: C \rightarrow X$ be defined by $f(x)=(x-2)(x-4) / 3$.

The function $f$ maps 2 and 4 to 0 , then it maps 0 inside $(2,4)$, and finally it maps the open interval $(2,4)$ outside $C$.

Therefore $X, C$, and $f$ satisfy the assumptions of Problem 2.1. Moreover $X$ is connected, $C$ is not connected, $C_{2}=\partial C=\{0,2,4\}, C_{3}=\{2,4\}$, and $C_{4}=\emptyset$. In particular $\operatorname{Iter}(f, C, X)=3$. 
Example 4.3 Let $X=\left\{(x, y) \in \mathbb{R}^{2}: x^{2}+y^{2}=1\right\}$ be the circle, which we parametrize as usually with the angles in $[0,2 \pi]$. Let $C:=[2 \pi / 5,8 \pi / 5]$ (namely $3 / 5$ of the circle), and let $f: C \rightarrow X$ be the counterclockwise rotation by $4 \pi / 5$ (namely $2 / 5$ of the way around the circle).

It turns out that $X, C$, and $f$ satisfy the assumptions of Problem 2.1 (in this case indeed $\partial C$ consists of the two points corresponding to $2 \pi / 5$ and $8 \pi / 5$ ). Moreover $X$ and $C$ are connected, and it is not difficult to see that $\operatorname{Iter}(f, C, X)=4$. The sets $C_{1}, \ldots, C_{4}$ are represented in the following picture.
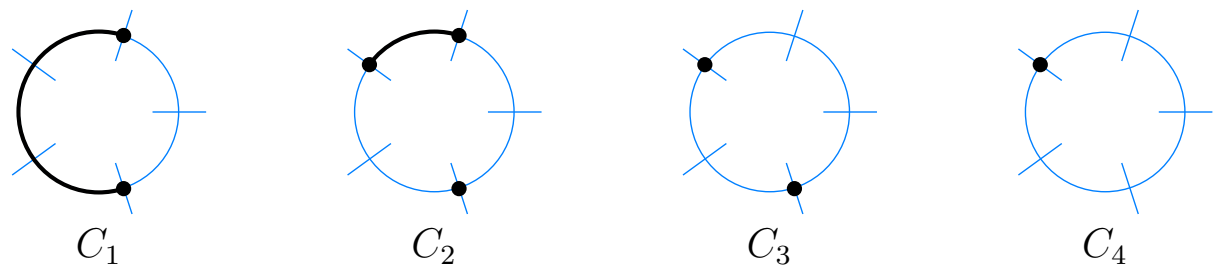

We can obviously replace 5 with any greater odd integer $d$. In this way we obtain a function which can be iterated exactly $d-1$ times.

Example 4.4 Let us consider the following subsets of the real plane:

$$
C:=\left\{(x, 0) \in \mathbb{R}^{2}: x \in \mathbb{R}\right\}, \quad X_{1}:=\bigcup_{k \in \mathbb{Z}}([5 k, 5 k+2] \times \mathbb{R}) .
$$

Let $X:=X_{1} \cup C$. Clearly both $X$ and $C$, with the topology inherited as subsets of $\mathbb{R}^{2}$, are connected, simply connected, contractible. Let $f: C \rightarrow X$ be defined by

$$
f(x, 0):= \begin{cases}(x+2,0) & \text { if } x \in[5 k, 5 k+3] \text { for some } k \in \mathbb{Z}, \\ (x+2,|5 k+4-x|-1) & \text { if } x \in[5 k+3,5 k+5] \text { for some } k \in \mathbb{Z} .\end{cases}
$$

Roughly speaking, $C$ is the $x$ axis, $X$ is the union of $C$ and some periodically arranged vertical stripes, $f$ is a translation by 2 in the $x$ direction followed by a vertical bending inside the stripes. The following picture shows the action of $f$ on some points of $C$.
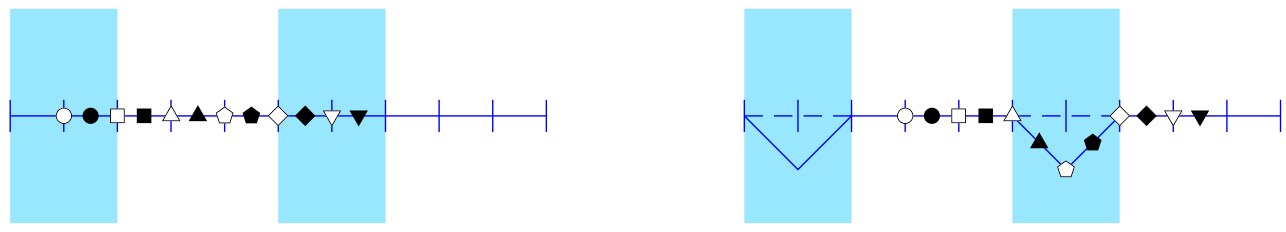

The boundary of $C$ in $X$ is the union of the segments of the form $[5 k, 5 k+2] \times\{0\}$ (the intersection of $C$ with the vertical stripes). The function $f$ just translates these segments in the $x$ direction, keeping them inside $C$. Therefore all the assumptions of 
Problem 2.1 are satisfied. It is not difficult to check that $\operatorname{Iter}(f, C, X)=5$, and the sets $C_{2}, \ldots, C_{5}$ are those represented in the following picture (we represent only one period, of course).
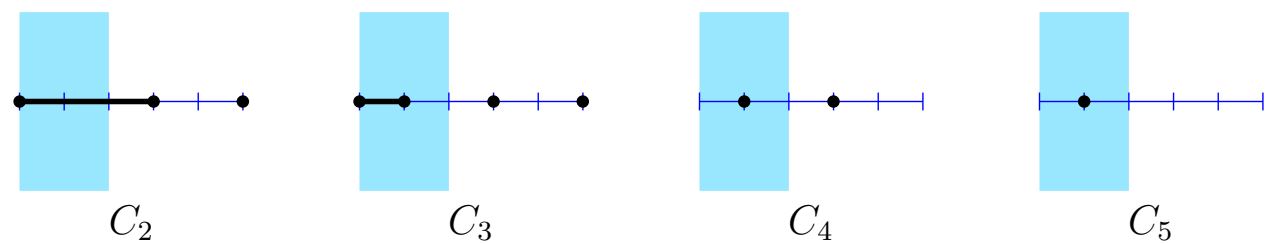

The function $f$ is also homotopic to the identity in the sense of Problem 2.2, since both are homotopic to the translation by 2 in the $x$ direction. Therefore also the assumptions of Problem 2.2 are satisfied, and this shows the optimality of the estimate of $\operatorname{Iter}(f, C, X)$ given in Theorem 2.8,

Note that in Example 4.4 above the set $C$ is not compact. At the present we have no example of a function $f: C \rightarrow X$ satisfying the assumptions of Problem 2.1 with $X$ simply connected, $C$ compact and connected, and $\operatorname{Iter}(f, C, X)=5$.

As we have seen, Example 4.4 above shows also the optimality of Theorem 2.8. We now give another example, in which the subset $C$ is not only closed, but also compact.

Example 4.5 Let us consider polar coordinates $(\rho, \theta)$ in the Euclidean plane. Let

$$
\begin{aligned}
& X:=\left\{(\rho \cos \theta, \rho \sin \theta) \in \mathbb{R}^{2}: 1 \leq \rho \leq 3, \theta \in[0,2 \pi]\right\}, \\
& Y_{1}:=\left\{(\rho \cos \theta, \rho \sin \theta) \in \mathbb{R}^{2}: 1 \leq \rho \leq 2, \theta \in[0,2 \pi]\right\}, \\
& Y_{2}:=\left\{(\rho \cos \theta, \rho \sin \theta) \in \mathbb{R}^{2}: 2 \leq \rho \leq 3,2 \pi / 5 \leq \theta \leq 8 \pi / 5\right\} .
\end{aligned}
$$

Let $C:=Y_{1} \cup Y_{2}$, and let $f: C \rightarrow X$ be the function represented in polar coordinates by

$$
(\rho, \theta) \rightarrow\left(\frac{5}{2}, \theta+\frac{4 \pi}{5}\right) .
$$

We claim that $X, C$, and $f$ satisfy the assumptions of Problem 2.2. Indeed, due to our choice of $X, \partial C$ contains only the arc with $\rho=2$ and $\theta \in[-2 \pi / 5,2 \pi / 5]$, and the two line segments with $\rho \in[2,3]$ and $\theta \in\{-2 \pi / 5,2 \pi / 5\}$. Therefore the function $f$ sends $\partial C$ in the points with $\rho=5 / 2$ and $\theta \in[2 \pi / 5,6 \pi / 5]$, hence inside $C$. As for the required homotopy, roughly speaking it can be constructed in three steps: reduction to the level $\rho=2$, rotation, reduction to the level $\rho=5 / 2$.

After the first iteration all points have radius equal to $5 / 2$, while with regard to the angle we have the identical situation of Example 4.3. It is now simple to see that Iter $(f, C, X)=5$ and the sets $C_{1}, \ldots, C_{5}$ are those represented in the following picture. 

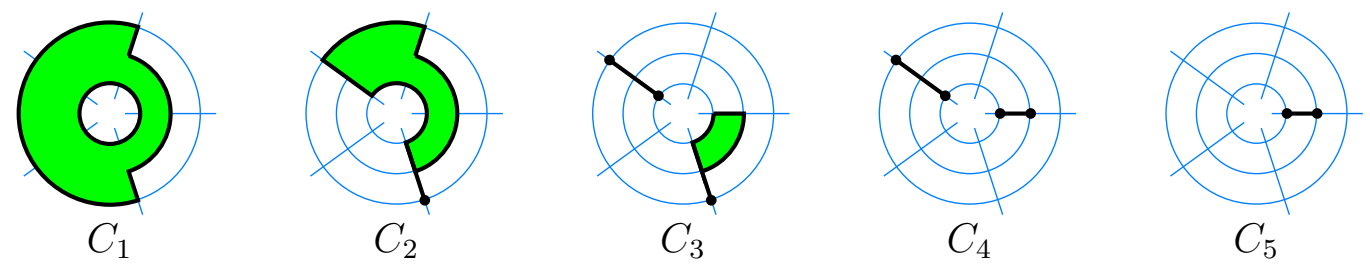

The following Example refers to the Euclidean case of Problem 1.1. It is probably the main example of this paper.

Example 4.6 Let $X:=\mathbb{R}^{2}$ be the Euclidean plane, and let $C$ be as in Example 4.5. Let $f: C \rightarrow X$ be represented in polar coordinates by

$$
(\rho, \theta) \rightarrow\left(\frac{5-|\rho-2|}{2}, \theta+\frac{4 \pi}{5}\right)
$$

It is clear that $X$ and $C$ are connected, and $X$ is simply connected. We claim that $X, C$, and $f$ satisfy the assumptions of Problem 2.1. In this case indeed $\partial C$ contains also the points in $C$ with $\rho=1$ and $\rho=3$, but the image of these points is contained in the level $\rho=2$, hence inside $C$. Moreover, the function $f$ is homotopic to the identity in the sense of Problem 2.2 (as in Example 4.5 the homotopy can be realized through the level $\rho=2$ ).

After two iterations all points have a radius strictly between 2 and 3, while with regard to the angle we have the identical situation of Example 4.3. It is not difficult to see that $\operatorname{Iter}(f, C, X)=6$ and the sets $C_{1}, \ldots, C_{6}$ are those represented in the following picture.
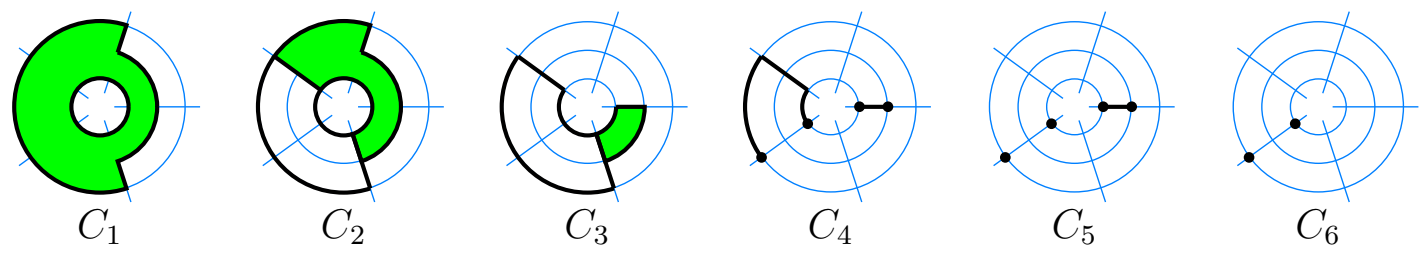

The constructions presented in Example 4.3 and Example 4.6 can be extended to higher dimensions, proving that also the triviality of some higher dimensional cohomology groups of $C$ and $X$ doesn't imply the existence of an infinite orbit.

Example 4.7 Let $X$ be the unit sphere in $\mathbb{R}^{2 n}=\mathbb{C}^{n}$, and let $d_{1}, \ldots, d_{n}$ be pairwise coprime odd integers each greater than or equal to 5 . Let us define $\lambda_{k}:=\exp \left(4 \pi i / d_{k}\right)$, and

$$
f\left(z_{1}, \ldots, z_{n}\right):=\left(\lambda_{1} z_{1}, \ldots, \lambda_{n} z_{n}\right) .
$$

Let $O$ be the set of points $\left(z_{1}, \ldots, z_{n}\right) \in X$ with $0<\arg \left(z_{k}\right)<4 \pi / d_{k}$ for every $k=1, \ldots, n$, and let $C:=X \backslash O$. 
For this choice it is clear that $C$ is contractible, $H^{i}(X)=0$ for all $1 \leq i \leq 2 n-1$, and $\operatorname{Iter}(f, C, X)=d_{1} d_{2} \cdots d_{n}$.

Again, one can improve the construction via the same process as in the passage from Example 4.3 to Example 4.6 to get a similar example with $X=\mathbb{R}^{2 n}$ and $H^{i}(C)=0$ for all $1 \leq i \leq 2 n-1$.

Now we present two examples concerning set-valued maps. The first one shows that without connectedness assumptions on the images only trivial iterations are guaranteed.

Example 4.8 Let $X:=\mathbb{R}$ with the usual topology, and let $C:=[0,4]$. Let $f: C \rightarrow$ $\mathcal{P}_{\star}(X)$ be defined by

$$
f(x):= \begin{cases}\{2\} & \text { if } x \in[0,1) \cup(3,4] \\ \{2,5\} & \text { if } x \in\{1,3\} \\ \{5\} & \text { if } x \in(1,3)\end{cases}
$$

It is easy to show that $X$ and $C$ are connected (and even contractible), $f$ satisfies (usc) and (Bdr-s), and $\operatorname{Iter}(f, C, X)=2$.

The following Example shows the optimality of statement (3) in Theorem 2.12.

Example 4.9 Let $X:=\mathbb{R}^{2}$ with the usual topology, let $Q$ be the square $[0,2] \times[0,2]$, let $S$ be the segment with endpoints $(2,0)$ and $(3,0)$, and let $C:=Q \cup S$. Let $d(x, y)$ denote the distance of the point $(x, y)$ from the boundary of $Q$. Let $f: C \rightarrow \mathcal{P}_{\star}(X)$ be defined in the following way:

- if $(x, y)=(2,0)$, then $f(x, y)$ is the segment with endpoints $(1,1)$ and $(3,0)$;

- if $(x, y) \in S \backslash Q$, then $f(x, y)$ is the singleton $\{(1,1)\}$;

- if $(x, y) \in Q \backslash S$, then $f(x, y)$ is the singleton $\{(3, d(x, y))\}$.

In a few words, $f$ is single-valued except at $(2,0)$ : it send $\partial Q \backslash S$ to $(3,0)$, in turn $(3,0)$ and the rest of $S \backslash Q$ are sent inside the square at $(1,1)$, and the interior of $Q$ is sent outside $C$. Finally, the image of $(2,0)$ is the minimal convex set for which the resulting function turns out to be upper semicontinuous.

Therefore $f$ satisfies (usc), (Bdr-w), (Conn). Moreover Iter $(f, C, X)=4$, and the sets $C_{1}, \ldots, C_{4}$ are those represented in the following picture.
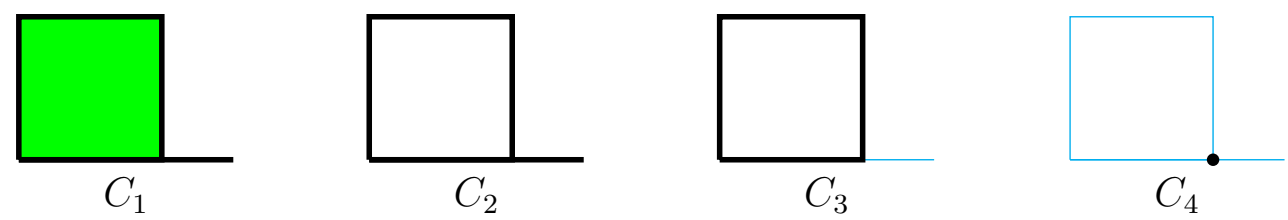


\section{$5 \quad$ Stochastic games and iterations}

Stochastic games In literature there are different and sometimes contradictory definitions of stochastic game, with different levels of generality. For the sake of simplicity, we present here a definition which is quite restrictive, but yet enough to describe this field and its open problems. In this paragraph we stick to the notations of [12].

A stochastic game is played by a finite set $P$ of players over a finite set $S$ of possible states, and involves a finite or countable number of stages. At each stage $n$ the game is in some state $s_{n} \in S$. If this is not the last stage of play, each player chooses an action in a finite set $A$ of possible options, and the state of the game changes to some $s_{n+1}$ which is a (possibly random) function of $s_{n}$ and of the element of $A^{P}$ representing the players' choices. Moreover, each player receives a payoff, which also depends on $s_{n}$ and on the actions selected. Then the game moves to next stage. In all stages, all players have complete knowledge of the past history of play, of the present state, and of the present options and their consequences. The only uncertainty concerns what the other players will do in the present and in the future.

Let $H_{n}:=S \times\left(S \times A^{P}\right)^{n-1}$ denote the set of possible histories up to stage $n$, and let $H$ denote the union of $H_{n}$ as $n$ varies over all stages, namely the set of all finite histories. A strategy for a given player is a function $H \rightarrow \operatorname{Prob}(A)$, where $\operatorname{Prob}(A)$ is the space of probability measures on $A$ (actually it is a simplex). When the game is in stage $n$, and $h_{n} \in H_{n}$ is the history up to that stage, then the value of the strategy in $h_{n}$ is the lottery used by the player in order to select next action.

Up to introducing a cumbersome notation, one could also admit that states are a set $S_{n}$ depending on the stage $n$, and possible actions are a set $A_{n, s, p}$ depending on the stage $n$, on the state $s$, and on the player $p$. Of course in this more general setting the definitions of histories and strategies need to be changed accordingly.

For any $\varepsilon \geq 0$, an $\varepsilon$-equilibrium in a game is a profile of strategies, one for each player, such that no player can gain in expected payoff by more than $\varepsilon$ by choosing a different strategy, given that all the other players do not change their strategies (for more precision, see [12]). An equilibrium is a 0-equilibrium. We say that approximate equilibria exist if there exists an $\varepsilon$-equilibrium for every $\varepsilon>0$.

It is well known that equilibria exist whenever the stochastic game has finitely many stages. This is a celebrated result by J. NAsH [6].

When there are infinitely many stages of play, things are more complex. In the special case where the number of players is two, N. VIEILLE [12, 13, 14] proved existence of approximate equilibria. With three or more players, it is not known whether all stochastic games have approximate equilibria. R. Aumann, during his Address to the first world congress of "The Game Theory Society" (GAMES 2000, Bilbao), stated that this question is the most important open problem of mathematical game theory today. 
Quitting games Quitting games are a special class of stochastic games with a very simple structure. At any stage of a quitting game, each player has only two actions, c for continue and $q$ for quit. As soon as one or more of the players at any stage chooses $\mathrm{q}$, the game stops, and players receive payoffs, which depend on the subset of players that choose simultaneously the action $\mathrm{q}$. Whenever all players choose $\mathrm{c}$, the game goes to the next stage, and all players receive the payoff of 0 for that stage. Quitting games were studied first by J. FlesCh, F. ThuiJsman and O. J. VRIEZE in [2, but modelled first in full generality by E. Solan and N. Vieille [10].

The complexity of quitting games lies in the potentially large number of players. In the case of two players, one can prove existence of stationary $\varepsilon$-equilibria, namely $\varepsilon$-equilibria where strategies depend on the past history only through the current state. In [2] a three-player example was shown, where $\varepsilon$-equilibrium strategies have a nonconstant cyclic structure. This motivated the study of the three-player case, solved by E. SOLAN [9] by proving that approximate equilibria do exist. With four or more players, the problem is still open.

In a few words, despite the simpler structure, quitting games are important for both the positive and negative sides of the question of whether approximate equilibria exist for general stochastic games.

Quitting games and iterations of set-valued maps An approach connecting quitting games and topological dynamics has been introduced by E. Solan and N. VIEILLE in [10]. We sketch the main steps of this approach following [10], to which we refer for further details.

The main idea is to break up the game into infinitely many one-shot games, namely games played in one stage only.

To this end, let $N:=|P|$ be the number of players. For every fixed vector $w \in \mathbb{R}^{N}$, let $\Gamma_{w}$ be the one-shot game where the payoff vector is the same as in the original quitting game if at least one player chooses $\mathrm{q}$, and it is $w$ otherwise. The strategy of a player in $\Gamma_{w}$ is just the probability to choose c, so that $[0,1]^{N}$ is the set of strategy profiles for $\Gamma_{w}$. Let $f(w, p) \in \mathbb{R}^{N}$ be the expected payoff vector in the game $\Gamma_{w}$ when all players perform according to some strategy profile $p=\left(p_{1}, \ldots, p_{N}\right) \in[0,1]^{N}$, and let $q(p):=1-p_{1} \cdot \ldots \cdot p_{N}$ be the probability that at least one player chooses $\mathrm{q}$.

Now let $\rho$ be a large enough constant, depending only upon payoff vectors, and let $\varepsilon>0$ be small enough. Let $E_{\rho \varepsilon}(w) \subseteq[0,1]^{N}$ be the set of $\rho \varepsilon$-equilibria for the game $\Gamma_{w}$, which is nonempty because of Nash's theorem, and let us finally define

$$
F_{\varepsilon}(w):=\left\{f(w, p): p \in E_{\rho \varepsilon}(w), q(p) \geq \varepsilon\right\} \subseteq \mathbb{R}^{N} .
$$

We have thus a map $F_{\varepsilon}$ from $\mathbb{R}^{N}$ to subsets of $\mathbb{R}^{N}$, which can be subjected to iteration. It is not difficult to see that this set-valued map is upper semicontinuous. The problem is that $F_{\varepsilon}(w)$ might be empty for a set of $w$ which is eventually reached by any iteration process, and this would prevent infinite orbits from existing. 
The main achievements of [10] are providing conditions on the game that ensure that an infinite orbit exists (Proposition 2.2), and proving that any orbit defines an equilibrium (Proposition 2.4). This relates equilibria of the game with dynamics.

Relations with DVT What is actually proved in Proposition 2.2 of [10] is that, under suitable conditions on the game, there exists a compact set $C \subseteq \mathbb{R}^{N}$ such that $F_{\varepsilon}(w) \cap C \neq \emptyset$ for every $w \in C$. This guarantees for free the existence of an infinite orbit, and produces a class of quitting games with approximate equilibria. The existence of such a set is however just a sufficient condition.

Further relations between games and dynamics have been investigated in [7, 8]. In these papers $F_{\varepsilon}(w)$ is defined as in (5.1), but without condition $q(p) \geq \varepsilon$, so that now $F_{\varepsilon}(w)$ is trivially nonempty for every $w$. With such a definition, Theorem 3 in [8] states that (but for trivial cases) approximate equilibria exist if and only if for every small enough $\varepsilon>0$ there exist (infinite) orbits of this new $F_{\varepsilon}$ with unbounded total variation (the total variation of an orbit is the sum of distances between consecutive terms). This is a necessary and sufficient condition. The requirement on the total variation, which in the game context implies eventual quitting with certainty, rules out fixed points, and makes the problem highly nontrivial.

Theorem 1 of [7] states that there exists a compact connected set $C \subseteq \mathbb{R}^{N}$ such that $F_{0}$, as a map form $C$ to $\mathcal{P}_{\star}\left(\mathbb{R}^{N}\right)$, has the following topological properties. The set of points $w \in C$ such that $w \in F_{0}(w)$ coincides with $\partial C$, and $F_{0}$ is homotopic to the identity map on $C$ through a homotopy whose intermediate maps keep again all points of $\partial C$ fixed. This homotopy condition motivated our interest in our Problem 2.2.

Several tricks can be devised in order to avoid useless stationary or converging orbits. One possibility is taking the set $C$ considered before, and introducing a new set-valued map $G_{\varepsilon}$ whose graph is obtained from the graph of $F_{\varepsilon}$ by removing an open set containing the fixed points $(w, w)$ with $w \in \partial C$. One can prove that, if $\varepsilon>0$ and the removed open set is small enough, this construction gives a well defined set-valued map without fixed points, and such that for every $w \in \partial C$ the image $G_{\varepsilon}(w)$ still contains some motion back to the set $C$. Due to the lack of fixed points, any orbit of $G_{\varepsilon}$ is a non-converging orbit of $F_{\varepsilon}$, hence an orbit with unbounded total variation. We have thus reduced the problem to a situation similar to our Problem 2.1 or Problem 2.9.

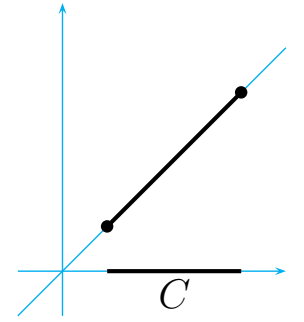

Identity on $C$

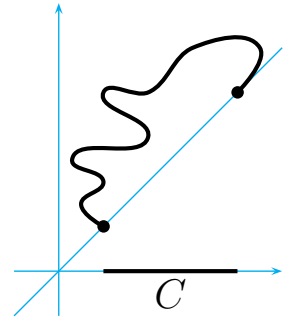

Graph of $F_{0}$

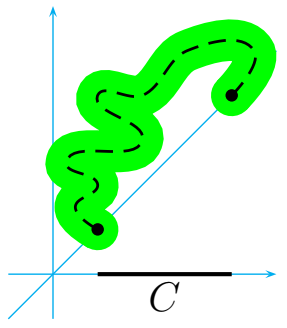

Graph of $F_{\varepsilon}$

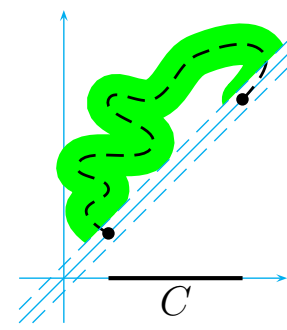

Graph of $G_{\varepsilon}$ 
The picture above is an attempt to represent this situation, with all limitations of a two dimensional setting.

Approaches of this kind have failed so far to establish the existence of approximate equilibria for quitting games, mostly due to the lack of any corresponding theorems of topological dynamics that demonstrates the existence of infinite orbits from some topological properties extracted from quitting games.

Indeed, on the game side we suspect that there are quitting games that do not have approximate equilibria, as well as on the dynamics side we suspect that there are many more plausible "results" concerning the existence of infinite orbits that fail to be true.

\section{Open problems}

As mentioned in the introduction, the following is probably the main question in Discrete Viability Theory.

Open Problem 6.1 Find nontrivial sufficient conditions on $f, C, X$ in order to have that $\operatorname{Iter}(f, C, X)=+\infty$.

Here "nontrivial" means that these conditions should be satisfied by reasonable classes of functions $f$ without fixed points and with $f(C) \nsubseteq \subset C$.

A first step in this direction could be to understand whether strengthening the topological assumptions on $f, C, X$ guarantees further iterations. This leads to the second question.

Open Problem 6.2 Under the assumptions of Problem 2.1 find intermediate results between statement (4) and statement (5) of Theorem 2.7.

Example 4.4 shows that 5 iterations is the most one can expect even when $C$ and $X$ are contractible, hence as simple as possible from the topological point of view. This seems to be the tombstone on the search of further iterations. Nevertheless, we point out once again that in that example $C$ is not compact. So a new frontier is understanding the role played by compactness in this subject, even in the simpler case.

Open Problem 6.3 Find the maximal number of iterations which are assured under the assumptions of Problem 1.1.

We know that this number is at least 5 and at most 6 . We also know that if this number is 6 it is a matter of compactness. If this is the case, then we can ask ourselves what happens with further topological requirements on $C$, for example if $\check{H}^{1}(C)=0$ (just to rule out Example 4.6). We know from Example 4.7 that we cannot expect an infinite orbit, but maybe the lower bound on the number of iterations increases.

Finally, a technical point for topologists.

Open Problem 6.4 Find the minimal assumptions on $Y$ under which Lemma 3.3 can be proved. 
Acknowledgments This research was supported financially by the German Science Foundation (Deutsche Forschungsgemeinschaft).

\section{References}

[1] J. P. Aubin; Viability Theory, Birkäuser, 1991.

[2] J. Flesch, F. Thuijsman and K. VRIEze, Cyclic Markov equilibria in stochastic games. Internat. J. Game Theory 26 (1997), no. 3, 303-314.

[3] S. T. Hu; Cohomology theory. Markham Publishing Co., Chicago, Ill.

[4] R. MCGEHEE; Attractors for closed relations on compact Hausdorff spaces. Indiana Univ. Math. J. 41 (1992), no. 4, 1165-1209.

[5] K. Mischaikow, M. Mrozek; Conley index. Handbook of dynamical systems, Vol. 2, 393-460, North-Holland, Amsterdam, 2002.

[6] J. F. NAsh; Equilibrium points in n-person games. Proc. Nat. Acad. Sci. U. S. A. 36, (1950), 48-49.

[7] R. Simon; A topological approach to quitting games. Math. Oper. Res., to appear.

[8] R. Simon; The structure of non-zero-sum stochastic games. Adv. in Appl. Math. 38 (2007), no. 1, 1-26.

[9] E. Solan; Three-player absorbing games. Math. Oper. Res. 24 (1999), no. 3, 669-698.

[10] E. Solan, N. Vieille; Quitting games. Math. Oper. Res. 26 (2001), no. 2, 265285.

[11] E. H. Spanier; Algebraic topology, Mc-Graw Hill, 1966.

[12] N. VieIlle; Two player stochastic games. I. A reduction. Israel J. Math. 119 (2000), 55-91.

[13] N. VieILlE; Two player stochastic games. II. The case of recursive games. Israel J. Math. 119 (2000), 93-126.

[14] N. VIEILlE; Small perturbations and stochastic games. Israel J. Math. 119 (2000), $127-142$.

[15] T. WiandT; personal communication. 